\title{
Effect of microwave assisted alkali pretreatment and other pretreatment methods on some properties of bamboo fibre reinforced cement composites
}

\author{
A. Banjo Akinyemi ${ }^{\text {a,* }}$, E. Temidayo Omoniyi ${ }^{\text {b }}$, Godwin Onuzulike $^{c}$ \\ ${ }^{a}$ Department of Agricultural and Biosystems Engineering, Landmark University, Nigeria \\ ${ }^{\mathrm{b}}$ Department of Wood Products Engineering, University of Ibadan, Nigeria \\ ${ }^{\mathrm{c}}$ Department of Civil Engineering, Landmark University, Nigeria
}

\section{H I G H L I G H T S}

- More cellulose extractives were removed through microwave irradiation.

- Microwave assisted alkaline treatment improved the fibre roughness and ductility.

- Cementitious composites with microwave treated fibers had optimum performance.

- Alkaline only treated fibers did not perform badly.

\section{A R T I C L E I N F O}

Article history:

Received 5 June 2019

Received in revised form 4 November 2019

Accepted 10 February 2020

Available online 18 February 2020

\section{Keywords:}

Microwave

Irradiation

Bamboo

Fibers

Strength

Properties

Cement

Treatment

Composite

\begin{abstract}
A B S T R A C T
This study investigated the effectiveness of microwave irradiation pre- treatment in combination with sodium hydroxide and other pretreatment methods on bamboo fiber cement composite characteristics. The materials used for this study include bamboo fibers, Portland cement, fine sand and calcium chloride. Microwave assisted sodium hydroxide (MT), sodium hydroxide alone (AA) and hot water (HT) treatments were used to pre-treat the bamboo fiber before its use in the cementitious composite. Mechanical tests were conducted after 7, 14 and 28 days while Fourier transformed Infrared spectroscopy (FTIR) with Scanning electron microscopy (SEM) and energy-dispersive X-ray spectroscopy (EDS) were used to characterise the composites. The use of microwave assisted alkali treatment is observed to have the best performance due to its more efficient role in improving fiber roughness, ductility and toughness in the cementitious composites.
\end{abstract}

(c) 2020 Elsevier Ltd. All rights reserved.

\section{Introduction}

Sustainable and green concrete technology could be developed through the use of natural fibers as reinforcement in cement composites. These fibers if adopted could be used to replace the conventional steel rebars and synthetic fibers which are more costly and less environment friendly [1]. Reported studies have shown that there are impressive results from mechanical, physical and thermal performance tests conducted on natural fiber reinforced cement composites. These studies include [2-4]. As good as this may sound, natural fibers are however bedeviled with some challenges which have hampered the adoption on a large scale in

\footnotetext{
* Corresponding author.

E-mail address: bantonbows@gmail.com (A.B. Akinyemi).
}

the construction industry. Among such setbacks are the problem of reduced workability [5], setting time [6], degradation in alkaline environment [7], mineralization [8] among others. Most studies are in agreement that these setbacks are caused by their hydrophilic nature which is as a result of the abundant presence of cellulose, hemicellulose, lignin and other extractives in their cell walls $[9,10]$. In other to mitigate this problem, several solutions have been proffered such as hot water treatment [11-13], alkaline pre-treatment $[14,15]$, acidic pre-treatment [16], polymer and silane coating [1719]. It should be noted that numerous researches exist on the use of microwave energy in assisting chemical treatment of biomass for different purposes such as to obtain feedstock for the production of bioenergy and green materials [20,21], pyrolysis for the production of liquid fuel [22] and production of char [23]. However, limited data exist on the use of microwave irradiation 
in natural fiber reinforced cement composite studies, one of such study considered its use in accelerated curing of coir fiber reinforced cement composites [24]. Microwave assisted chemical treatment is reported to have a higher efficiency in removing lignin, cellulose and other extractives in any biomass material more than using the chemical treatment method alone in isolation [25-27]. On application of microwave to aid the treatment of any biomass material, it interacts with the hydrogen bonds located in the cellulose (Fig. 1) thereby increasing the temperature and subsequently leading to breakage of the crystalline bonds in the cellulose. The disruptions of the crystalline bonds cause rapid movement and polarization of chemical groups in the molecule and the eventual reduction of the cellulose within the biomass. It has also been reported that it has the capacity to remove lignin as well from biomass materials because of its low polarity [28]. In another study, it was observed that $80 \%$ and $90 \%$ of hemicellulose and lignin were removed through application of short times of microwave irradiation treatment on cellulose based material [29].

It is therefore proposed in this study to investigate the role of microwave assisted sodium hydroxide chemical treatment in improving some selected properties of bamboo fiber reinforced cement composites. In order to ascertain the efficacy of this treatment, other established pre-treatment methods of hot water immersion and alkaline chemical treatment were also used and their properties compared.

\section{Experimental program}

\subsection{Materials}

Bamboo culms from the Bambusa vulgaris species of 4 to 6 years of age were cut and allowed to dry in the open for four weeks. The nodes were broken to obtain the strips which were cut into an average length of $20 \mathrm{~mm}$ before hammer milling was performed. Bamboo fibers passing $2.0 \mathrm{~mm}$ sieve was used. The fibers had fiber length of $1.82 \mathrm{~mm}$ and a fiber width of $11.08 \mu \mathrm{m}$. A general use type of Portland cement with 42.5 rating and fine aggregate from river sand having fineness modulus of 2.67 were used for the cement composite. $\mathrm{CaCl}$ of $3 \%$ (w/w of cement) was used as cement accelerator [31]. The chemical composition of the bamboo specie is shown in Table 1.

\subsection{Surface treatments of bamboo fibers}

Microwave irradiation assisted alkaline treatment (MT) was conducted using a domestic LG microwave oven MWO 2595 model with maximum power of $1000 \mathrm{~W}$ set to medium-low level of 300 Watts. The bamboo fibers were placed in glass vessel containing
Table 1

Proximate chemical composition of Bambusa vulgaris [32].

\begin{tabular}{ll}
\hline Chemical composition & $\%$ \\
\hline Lignin & 22.7 \\
Holocellulose & 77.4 \\
Alpha-cellulose & 55.4 \\
Cold water solubles & 8.5 \\
Hot water solubles & 10.4 \\
\hline
\end{tabular}

$10 \%$ concentration of sodium hydroxide which had been diluted with distilled water and subjected to microwave energy for $1 \mathrm{~h}$. At every interval of $10 \mathrm{~min}$, the microwave was stopped, and the container taken out and stirred for a few seconds. This is to ensure uniform heating within the container and this was done for the entire duration of heating [33]. Thereafter, the fiber was washed thoroughly in water for the chemical to leach out before it was dried in an oven at a temperature of $30^{\circ} \mathrm{C}$ until the change in consecutive moisture content readings of \pm 2 were achieved. For alkaline treatment only (AA), the bamboo fibers were immersed in water containing the same $10 \%$ conc. of $\mathrm{NaoH}$ which had been diluted with distilled water for $24 \mathrm{~h}$. Subsequently, the fibers were washed in water to remove the alkali absorbed on the surface and thereafter dried in the oven at the same temperature. Hot water treatment (HT) was performed by soaking the fibers in an aluminium pressure pot of $90 \mathrm{~L}$ containing hot water of $90^{\circ} \mathrm{C}$ and sealed for $1 \mathrm{hr}$ before it was decanted. The fibers were washed in $20 \mathrm{~L}$ of water at $22^{\circ} \mathrm{C}$ before oven drying at $35^{\circ} \mathrm{C}$.

\subsection{Preparation of treated fiber cement composites}

For the production of the composites, the mix design in Table 2 was used. Portland cement was mixed with sand in the ratio 1:2 while water : binder ratio was kept at 0.52 . The fibers were varied at 1 and $1.5 \%$ of the mass of the constituents and the entire mixture stirred manually until an homogenous mixture was attained. Two different moulds of $200 \times 150 \mathrm{~mm}$ for tensile test and $10 \times 400 \times 400 \mathrm{~mm}$ for bending strength tests were filled with the mixture while a total of 124 specimens were produced, compacted and cured for $24 \mathrm{~h}$ in the laboratory at ambient temperature. Thereafter, the specimens were demoulded and cured in water for 7, 14 and 28 days before the mechanical tests were conducted.

\subsection{Equipments}

\subsubsection{Fourier-transform infrared spectroscopy (FTIR) analysis}

FTIR spectra were used to evaluate the infrared spectrum of absorption of the fibers after treatment and fractured samples after
microwave inactive region
microwave active region
microwave inactive region

Fig. 1. Microwave relationship with cellulose due to existence of hydrogen bond [30]. 
Table 2

Experimental mix.

\begin{tabular}{|c|c|c|c|c|c|c|}
\hline Sample & Treatment & Time $(\mathrm{h})$ & Fibers (\%) & Sand (kg) & Cement (kg) & Replicates \\
\hline G1 & Hot water & 24 & 1 & 0.101 & 0.050 & 24 \\
\hline G2 & Hot water & 24 & 1.5 & 0.151 & 0.076 & 24 \\
\hline G3 & Microwave & 1 & 1 & 0.101 & 0.050 & 24 \\
\hline G4 & Microwave & 1 & 1.5 & 0.151 & 0.076 & 24 \\
\hline G5 & Alkali & 1 & 1 & 0.101 & 0.050 & 24 \\
\hline G6 & Alkali & 1 & 1.5 & 0.151 & 0.076 & 24 \\
\hline
\end{tabular}

the mechanical tests using a Shimadzu FTIR-8400S spectrophotometer. The spectra for the specimens were recorded by grinding the specimens to powder, mixing the powder with a small amount of potassium bromide powder and compacting the mixture into a disk.

\subsubsection{Scanning electron microscope and energy dispersive spectroscopy (SEM/EDS)}

Microstructural analysis was performed using a field emission scanning electron microscope (FESEM) (model:JSM-7600F, Jeol, Japan) at an acceleration potential of $15 \mathrm{kV}$ and a magnification of different magnifications. Fractured sample surfaces were sputter coated with a thin layer of platinum using a JFC-1600 auto-fine coater and thereafter examined.

\subsubsection{Universal testing machine}

Tensile and bending strength tests were performed using a Testometric Universal Testing Machine (Model No M500-50AT) using a cross head speed of $10 \mathrm{~mm} / \mathrm{min}$ for the tensile tests and loading rate of $0.5 \mathrm{~mm} / \mathrm{min}$ for the bending strength tests. Fig. 2 shows the load-deflection curves of some tested samples. There are three noticeable stages of pattern during the test relating to the damage level of the bamboo fiber composites. All the curves showed linear and non-linear phases in Fig. 2. The linear part is the elastic part before cracking occurred. In this stage the fibers and the cement matrix carry the load together. The next phase is where the real work of the fibers was noticed in which bridging of cracks was performed. This is termed the work stage in which G3 and G1 were able to carry the tensile stress more efficiently than the G1. The last stage is denoted for the phase in which the composite panel base starts to crack until failure occurred.

\subsection{Testing procedure}

Five specimens of $100 \times 50 \times 10 \mathrm{~mm}$ sizes were cut from the bending strength test samples for each composition and tested for modulus of elasticity (MOE) and modulus of rupture (MOR) after 7, 14 and 28 days water curing using the Testometric Universal Testing Machine. The mean values were used to represent the bending strength results. The split tensile test was also conducted after each successive curing duration with the same equipment at the specified loading rates. FTIR analysis was performed on the treated fibers after the treatments and also on fractured samples of fiber reinforced cement composites after mechanical tests. SEM-EDS analysis was conducted on some specimens from the bamboo fiber reinforced cement composites.

\section{Results and discussions}

\subsection{FTIR analysis of pre-treated bamboo fibers}

The characteristics of MT, AA and HT in Fig. 3 showed that there was a reduction in the peak at $3400.83 \mathrm{~cm}^{-1}$ for MT relative to peaks at wave numbers corresponding to AA and HT. This meant that using alkali treatment reduces hydrogen bonds in the hydro- xyl group but after irradiation with microwave more of these bonds were reduced than the other pre-treatment methods. The spectrum at this range is denoted for hemicellulose and fatty acid [34]. The transmittance at $2897.18 \mathrm{~cm}^{-1}$ (MT), $2924.18 \mathrm{~cm}^{-1}$ (AA) and $2926.11 \mathrm{~cm}^{-1}$ (HT) are attributed to $\mathrm{CH}$ and $\mathrm{CH}_{2}$ groups of fats, waxes and pectins [35]. It was observed that on application of MT treatment the least intensity in the extractives was observed translating to a reduction in this group; this was followed by a higher peak at AA and lastly the highest peak at HT. The peaks between 1737.92 and $1791.93 \mathrm{~cm}^{-1}$ disappeared upon microwave pretreatment which meant that the carboxylic group had been removed at MT but were still present at the AA and HT treated fibers. This group denotes the presence of non-cellulosic fibres such as pectin, lignin and hemicellulose [36]. The reduction of peak intensity at $1639.55 \mathrm{~cm}^{-1}$ signifies that $\mathrm{C}=\mathrm{C}$ which constitutes the lignin portion of the fibre had been reduced at MT and HT pretreated samples. The decrease in the sharp intensities between 1420 and $1456.36 \mathrm{~cm}^{-1}$ most especially the peak at $1423.51 \mathrm{~cm}^{-1}$ which is ascribed to $\mathrm{C}-\mathrm{H}$ bending in aromatic rings present majorly in lignin groups suggests the significant reduction of lignin by MT pre-treatment [37,38]. Table 3 gives a summary of the various functional groups of MT, AA and HT pre-treated fibre samples.

\subsubsection{FTIR analysis of bamboo fibers reinforced cement composites}

Some chemical changes took place after mixing the treated fibers with cement mortars after the curing had been stopped. This is shown in Fig. 4 and Table 4 respectively. The peak at the bands ranging from $3423.76-3441.12 \mathrm{~cm}^{-1}$ indicated that the blending of Portland cement with the fibers caused an increase in the peak intensity obtained prior to this. This difference was instituted by the $\mathrm{Ca}(\mathrm{OH})_{2}$ ascribed to $\mathrm{OH}$ band which shows that the chemical elements within the cement led to this change [39]. The incorporation of cement with the bamboo fibers caused the broadening of the bands at this range more than the IR peak at this band from ordinary fibers. This band is denoted for hemicellulose of $\mathrm{O}-\mathrm{H}$ groups [40]. Spectra bands at 2926.11, 2924.18 and $2928.04 \mathrm{~cm}^{-1}$ had a reduced intensity which is caused by the blending with cement, therefore the methylene and methyl vibration frequencies were overcome by the chemical constituents of the binder [41]. The IR peak of $1791.93 \mathrm{~cm}^{-1}$ from the ordinary fibers disappeared on mixing with cement particles but surfaced in the form of broad band at $1737.92 \mathrm{~cm}^{-1}$. Some bands such as $2127.55,1506.46,1464.02,1266.35,896.93 \mathrm{~cm}^{-1}$ did not appear because of inclusion of cement. The use of microwave energy to assist in the treatment of the fibers exposed the cellulose to more aggressive deposition of cement hydrates because of its increased surface area and ultimately a better bond was established between the fibers and the cement

\subsection{Mechanical results of bamboo fibre reinforced cement composites}

\subsubsection{Split tensile}

As shown in Fig. 5, MT had values ranging from 13.71 to $17.44 \mathrm{MPa}$ and 13.88 - $19.01 \mathrm{MPa}$ for 1 and $1.5 \%$ fibre inclusion. 


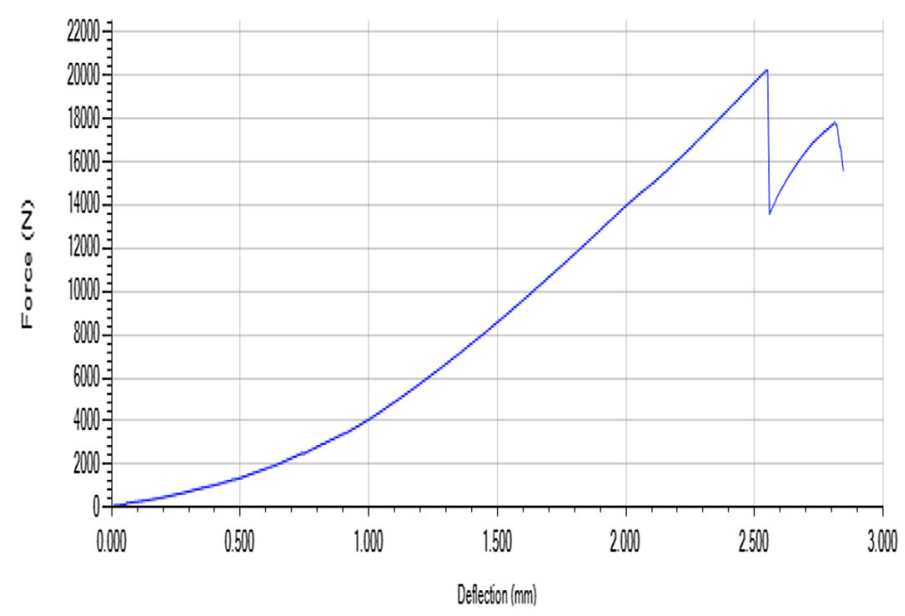

G1

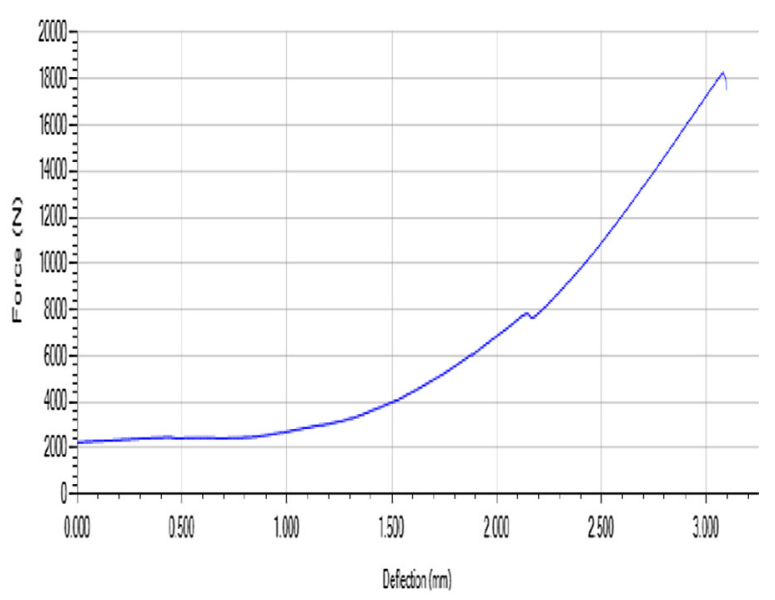

G3

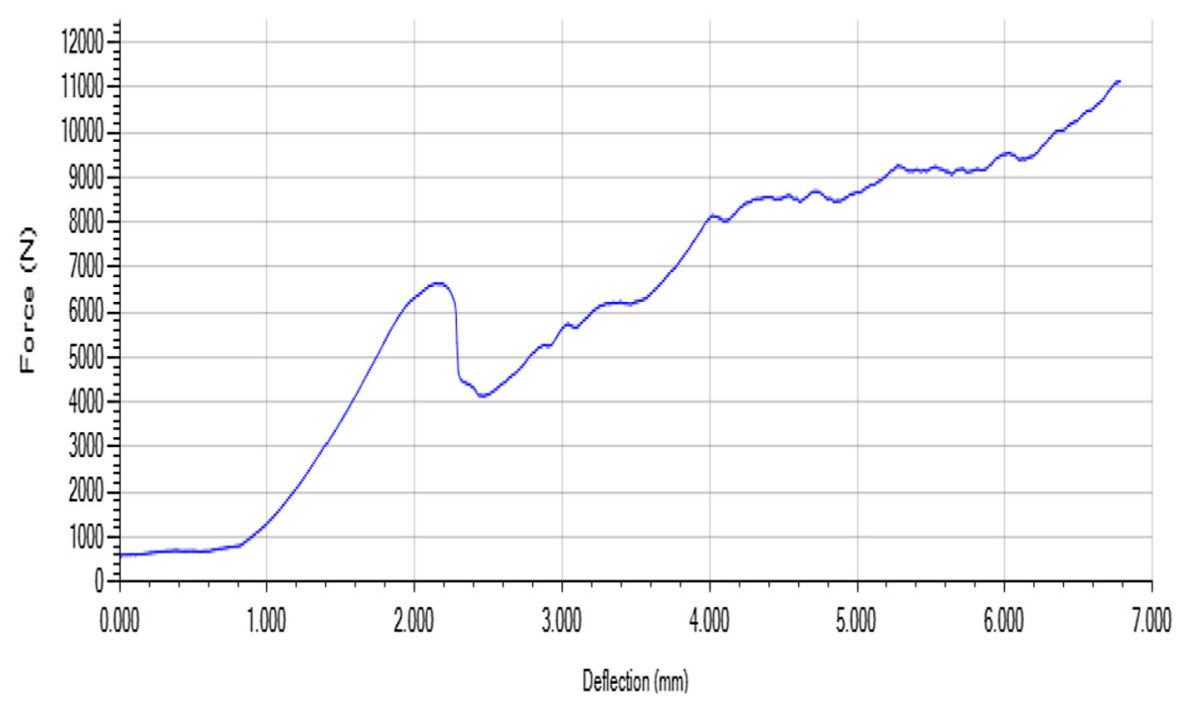

G6

Fig. 2. Load-deflection curves of cement composites.

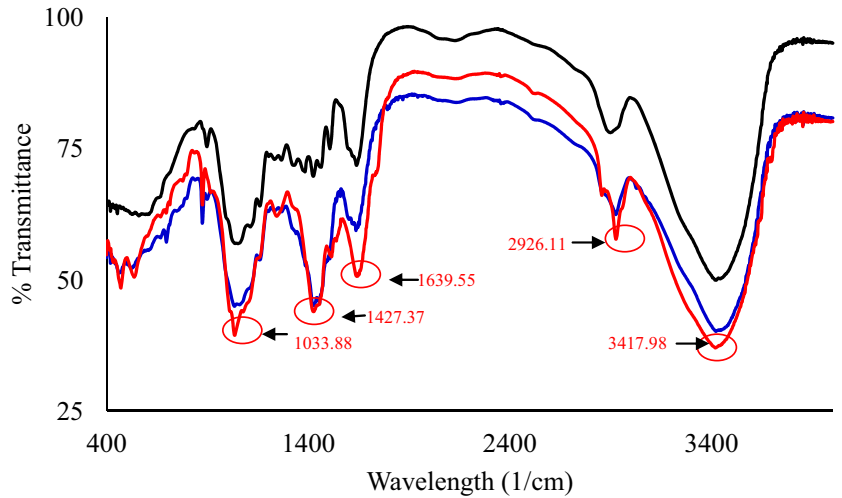

$\longrightarrow \mathrm{AA}-\mathrm{HT} \longrightarrow \mathrm{MT}$

Fig. 3. FTIR spectra of ordinary pre-treated bamboo fibers.
Table 3

FTIR bands of ordinary bamboo fibers.

\begin{tabular}{llll}
\hline Bond type & $\begin{array}{l}\text { Microwave } \\
\text { assisted }\left(\mathrm{cm}^{-1}\right)\end{array}$ & $\begin{array}{l}\text { Alkali alone } \\
\left(\mathrm{cm}^{-1}\right)\end{array}$ & $\begin{array}{l}\text { Hot water } \\
\left(\mathrm{cm}^{-1}\right)\end{array}$ \\
\hline -OH stretching & 3400.83 & 3411.83 & 3417.98 \\
C-H vibration & 2897.18 & 2924.18 & 2926.11 \\
C = H vibration & - & 1791.93 & 1737.92 \\
C = C stretching & 1639.55 & 1643.21 & 1639.55 \\
C-H bending & 1423.51 & 1456.30 & 1452.45 \\
C-C stretching & 1035.81 & 1058.96 & 1076.32 \\
\hline
\end{tabular}

AA treated fibers ranged from 11.06 to $12.76 \mathrm{MPa}$ and 11.95 - $15.66 \mathrm{MPa}$ at the same fibre variations. Similarly, HT samples ranged from $10.29-13.69 \mathrm{MPa}$ and $11.92-12.94 \mathrm{MPa}$ at 1 and $1.5 \%$ bamboo fibre inclusions. It was observed that MT treated fibres in the reinforced cement composites performed better as compared to AA and HT treated samples. This is because the fibers 


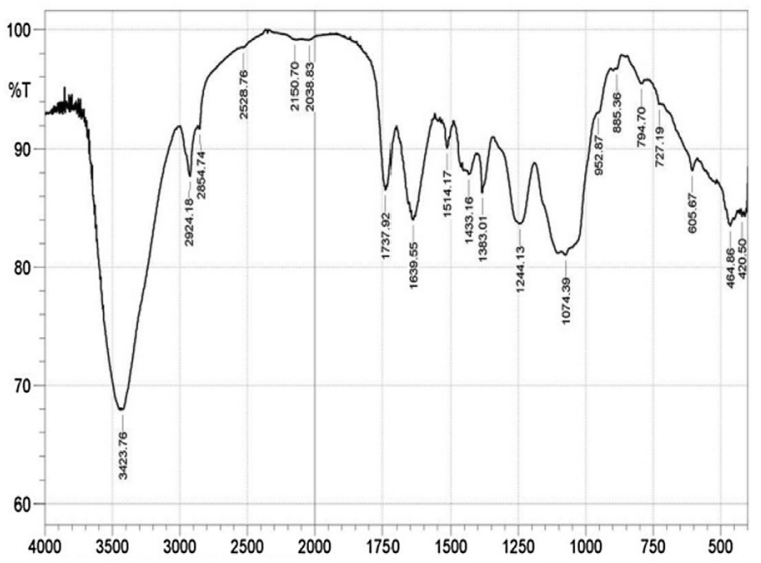

(a)

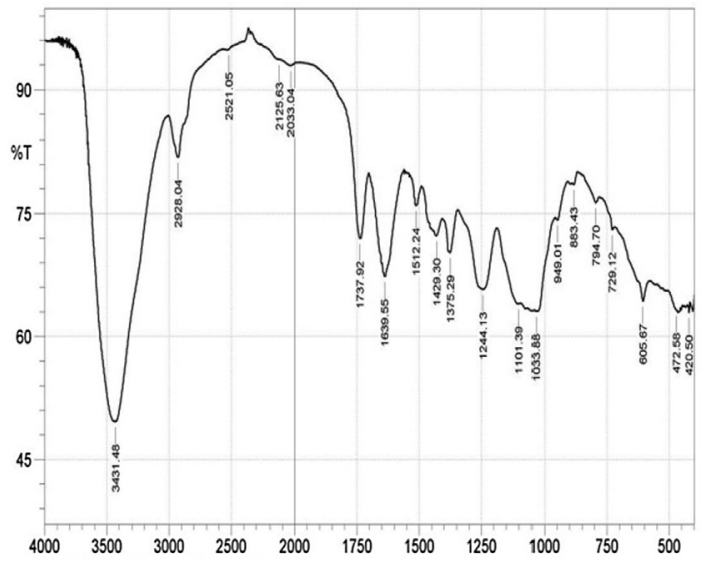

(b)

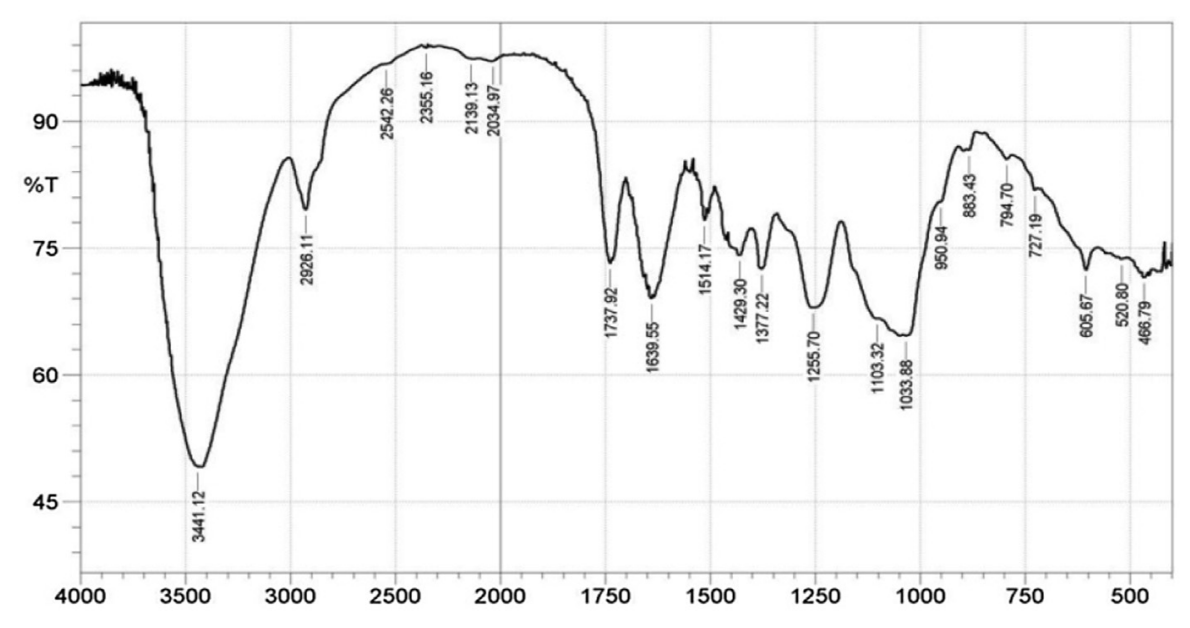

(c)

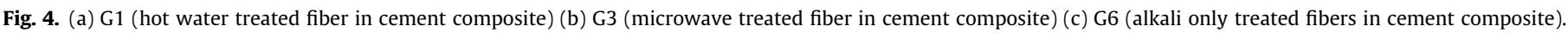

Table 4

Summary of FTIR spectra of microwave assisted alkaline, hot water and alkaline only treatments for bamboo fiber reinforcement in cement composites.

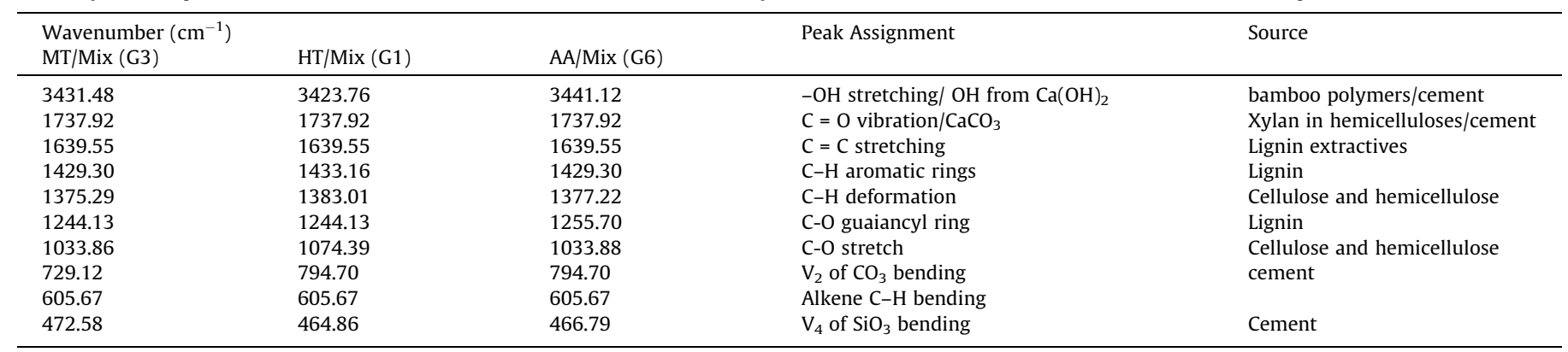

are more flexible and stronger after this treatment. Also, MT was able to roughen the surface of the fibers more than the other treatment methods thereby improving the strength and fibre-matrix bond at the interface. This was possible because the chemistry of the bamboo fibers had been altered due to more reduction in the hydroxyl groups which comprise of cellulose and lignin, hence an improvement in the interaction between the fibers and the matrix which subsequently led to this improved performance than the other treatments [42]. It had also been reported that on removal of hydrophilic constituents of cellulose fibers which are the hemicellulose, waxes and silica bodies after alkali treatment, the tensile properties of composites would be enhanced. This removal is only partial therefore many of the cellulose components are left open which affect the fibrils and helps to wet the fibers easily $[43,44]$. The tensile results for MT showed 24.32 and 20\% higher strength than HT and AA respectively while a slight improvement of AA over 


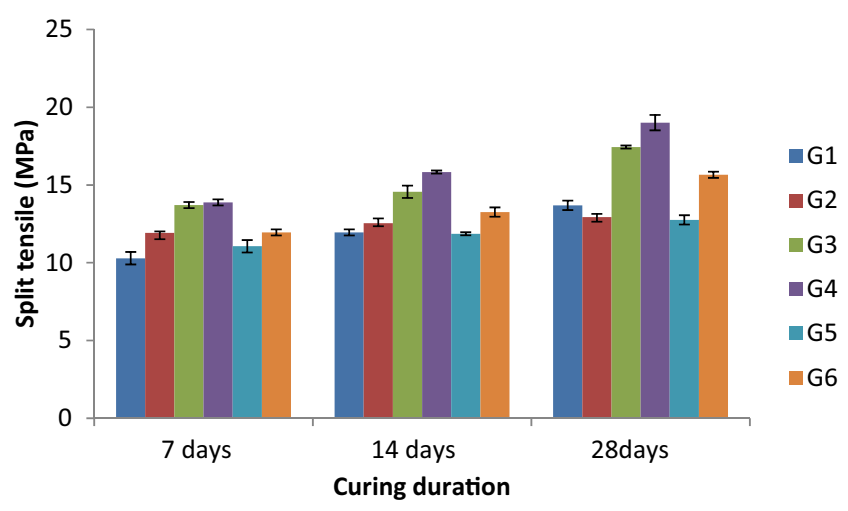

Fig. 5. Split tensile of samples. (Note: $G 1=H T, 1 \%$ fibre; $G 2=H T, 1.5$ fiber; $G 3=M T$, $1 \%$ fiber; G4 = MT,1.5\% fibre; G5 = AA, 1\% fiber; G6 = AA, $1.5 \%$ fiber).

HT was noted at $3.6 \%$ improved tensile strength. This meant that on treatment with AA some of the cellulose contents were removed thereby creating voids on the surface of the fibers which were filled by the cement hydration products leading to better tensile performance than HT treated samples which could possibly have very little deposition of the hydration products on the surface of the fibers due to minimal reduction of these extractives. But in the case of MT, more voids and pores were created on the fiber surface which translate to more deposition of cement hydration products on the fibre surface and ultimately enhanced the fibre-cement matrix adhesion caused by the mechanical interlocking mechanism $[45,46]$. The improved tensile properties from the microwave irradiated fibers in the cement composite samples are caused by the intense frequency of the dielectric system during the pretreatment which causes the chemicals to diffuse into the molecules of the fibre. This leads to agitation within the molecular structure and subsequently, the fibers rub against each other generating friction by reason of the electric field produced by the microwave energy. Uniform heat is then produced all over the entire surface area of the fibers and it aids greater penetration of the chemicals into the fibre structure without destroying the morphology [47].

\subsubsection{Modulus of rupture and elasticity}

The modulus of rupture and elasticity (MOR, MOE) displayed in Figs. 6 and 7 showed that MT treated fibers in the cement composites performed best in comparison with AA and HT. For MT, MOR improvement of 12,29 and $10.1 \%$ in relation to AA was observed while for MOE an increment of 4.9, 13.6 and 9.5\% in relation to AA was similarly noted after the curing duration. An higher difference in the bending strength was however observed on comparison between MT and HT with increase in flexural capacity of MT ranging from 54.5 to $97 \%$ in the MOR results and $16-59.1 \%$ for the MOE. One major reason for this improvement is that, MT fibers within the cement composites had the highest aspect ratio than both AA and HT treated samples. This was possible because an enhanced toughness had been induced in the fibers from the microwave irradiation in combination with the chemical treatment leading to the increase in aspect ratio which subsequently improved the ability of the fibers to bridge the cracks more efficiently than the other fibers [48]. It has been reported that combination of treatments are more effective in reducing the extractives in cellulose fibers than using a single treatment because such treatments lead to disintegration of large bundles of fiber structure causing an improvement in the fibrillated morphology [15]. The bending strength showed clear increase when the content of the bamboo fibers were increased from 1 to $1.5 \%$. The poor performance in bending strength shown by HT treated samples could possibly be caused by over removal of the extractives which ulti-

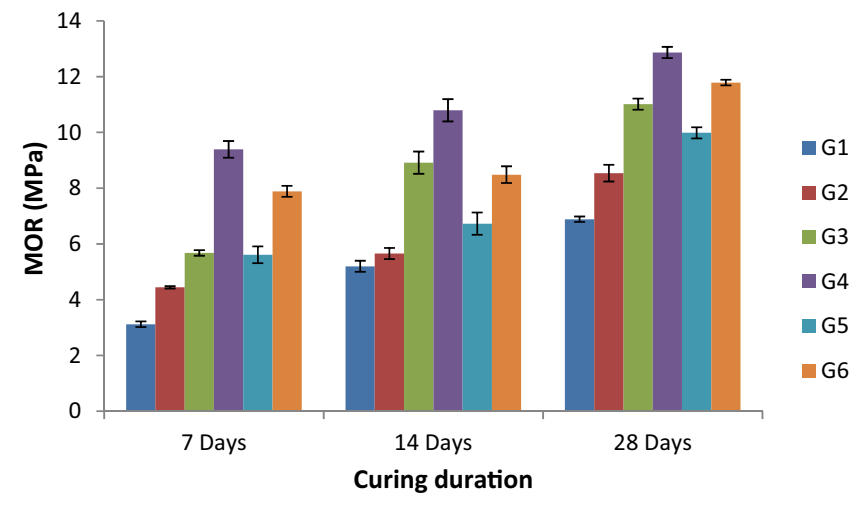

Fig. 6. Modulus of rupture of specimens.

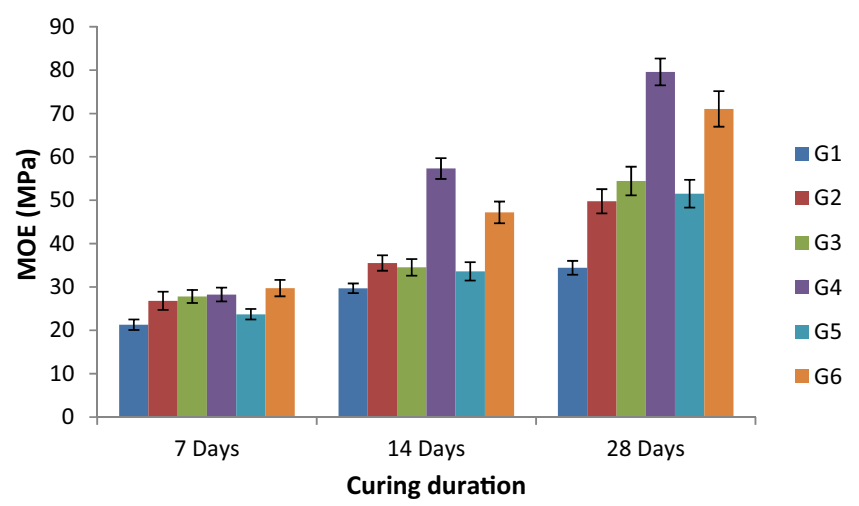

Fig. 7. Modulus of elasticity of specimens. (Note: G1 = HT, $1 \%$ fibre; G2 = HT, 1.5 fiber; G3 = MT, 1\% fiber; G4 = MT, 1.5\% fibre; G5 = AA, 1\% fiber; G6 = AA, 1.5\% fiber).

mately weakened the fibers, hence the poor resistance to rupturing caused by the fibers low tensile strength [49].

\subsection{Characterisation of bamboo fibre cement composites}

\subsubsection{Scanning electron microscope (SEM)}

The SEM micrographs are shown in Figs. 8, 9 and 10. Fig. 9 showed very distinct and clear interface between the hot water treated fibers and the mortar. But Figs. 8 and 10 indicate that there was homogenous blending between the alkali only treated fibers and microwave assisted alkali treated fibers with the cement mortars and therefore this makes the interface to be unclear. This could be credited to the fact that AA and MT pre-treatment methods produced rougher surface texture on the bamboo fibers than the HT pre-treated fibers and it produced better adhesion to the mortar. This was evident from the split tensile, modulus of rupture and elasticity results in which both had better performance than the hot water treated fibre reinforced cement composite samples. A similar statement was made by [50] that good adhesion between fibers and matrix produce high strength brittle material likewise a reversal is the case if a poor bonding is sighted at the interface. Numerous cracks were also found on the surface of Fig. 9 which ultimately affected its mechanical strength. However, fewer cracks occurred on the interface in Fig. 8 which translates to improved performance more than that of Fig. 8. Fig. 10 had minimal occurrence of cracks at the interface and so this gave it the best performance in the mechanical tests conducted. As shown in Figs. 8 and 10 , the fractured surface showed occurrence of fibre pull out and pores left by remnants of the pulled out fibers sticking out from the fractured sample surface. This implies that chemical treated 

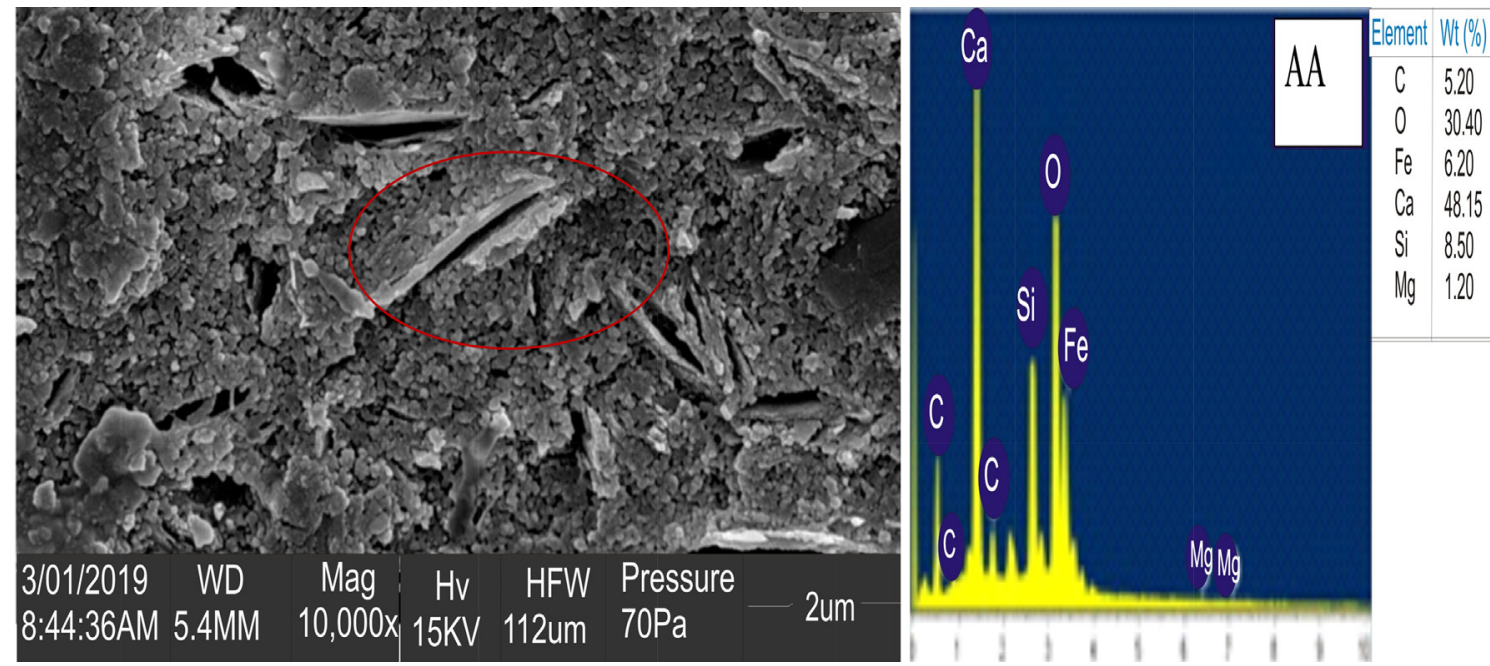

Fig. 8. SEM and EDS of AA treated fibers with mortar (marked portion is the interface).
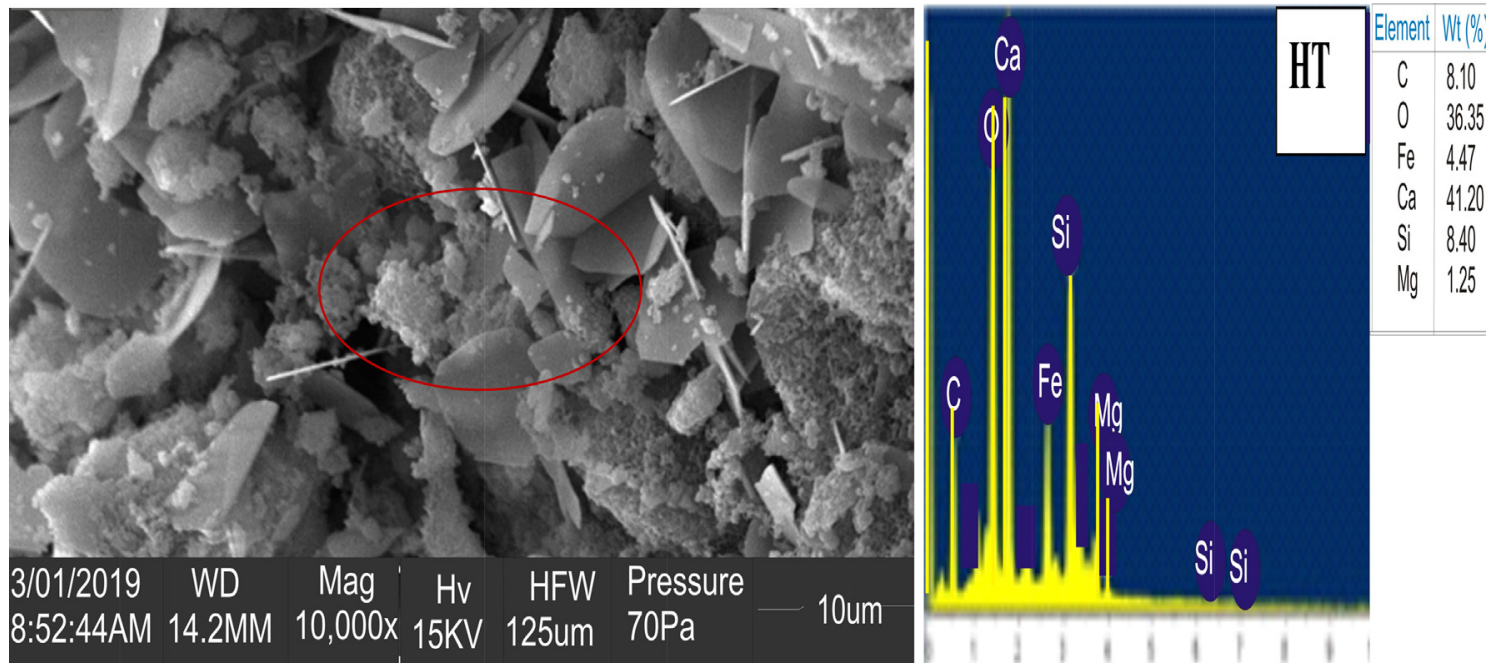

Fig. 9. SEM and EDS of HT treated fibers with mortar (marked portion is the interface).
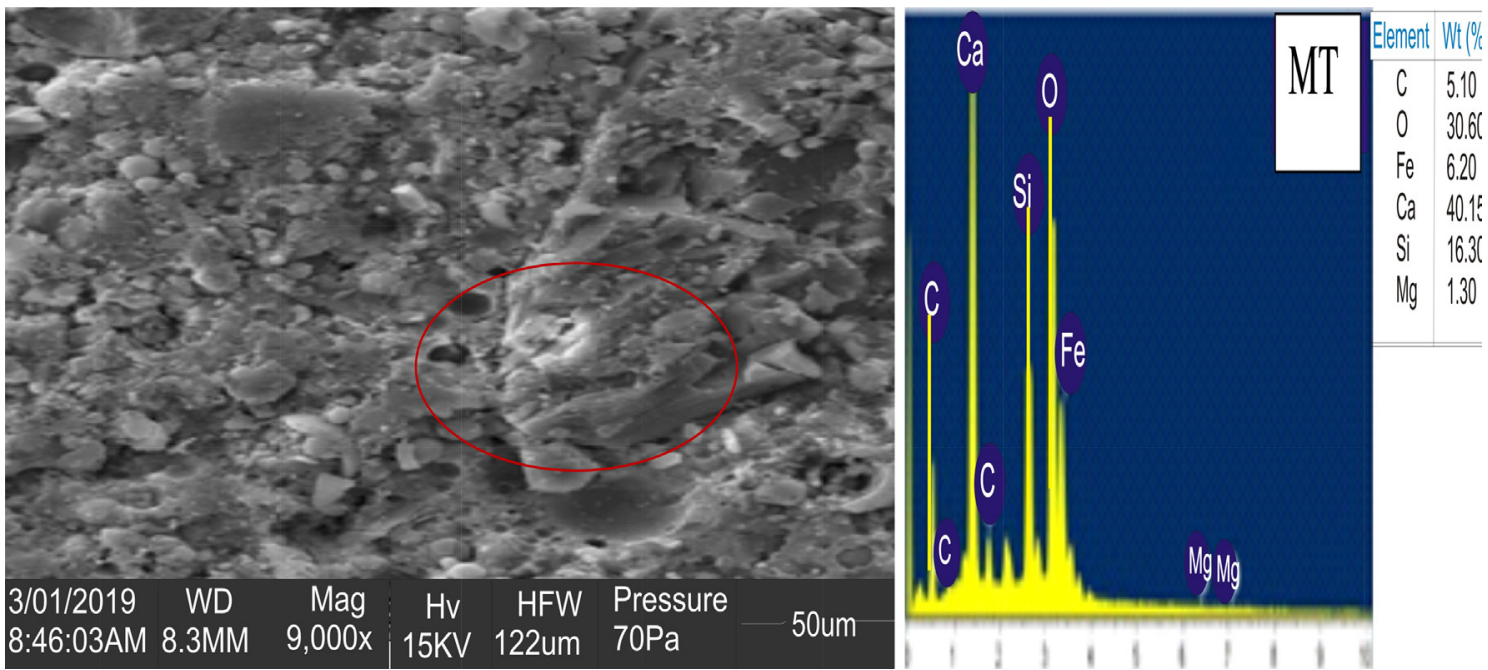

Fig. 10. SEM and EDS of MT treated fibers with mortar (marked portion is the interface). 
bamboo fibers has a better pull-out resistance from the cement composite because of better length to diameter ratio than the hot water treated fibers. Enhancement in absorption and energy dissipation during facture is the result of this resistance which hinders micro-crack formation and propagation, supports better and improved fiber toughness and stiffness in the cementitious matrix because of efficient stress transfer in the composite system. A previous study has shown that micro-fibers hinder the commencement of cracks; but macro-fibers however provide an efficient bridging and convey toughness with ductility to the fibre composites [51]. More cement hydration products could be observed on the micrograph surface in Fig. 9 which is due to the strong adhesion between the fibrillated bamboo fibers as seen from the FTIR results and the cement mortar which possibly improved the fibre-matrix bonding, hence the superior strength at the MT treated fibre cement composites. It had also been reported that better bonding occur between sodium hydroxide treated fibers and cement matrix due to precipitation of cement hydrated products into the fibrillated exposed cavities of the natural fibre [52]. A better adhesion is formed between the cellulose molecular structure and the calcium content of the cement hydration products

\subsubsection{Energy dispersive X-Ray (EDS)}

Elemental composition were done on some of the samples using EDS by selecting region of interest, however quantification error could occur as the sample volume is small usually in small micrometers. Nonetheless, the results show the occurrence of chemical elements with known chemical structures. MT treated fibre cement composites (Fig. 10) had the least proportion of calcium and chloride which constitute a major part of the cellulose, lignin and hemicellulose. This meant that the microwave irradiation was more effective in reducing its occurrence in the composite sample. AA treated fibre cement composites in Fig. 8 also had similar reduction in chloride content when compared with hot water treated fibre cement composites in Fig. 8. Similarly, the carbon/oxygen ratio for Figs. 8, 9 and 10 are $0.17,0.22$ and 0.16 respectively while the theoretical value based on chemical formular is 0.9 for cellulose and 2.9 for lignin [53]. This implies that the cement matrix samples containing microwave assisted alkali treated fibers had the least composition of both cellulose and lignin, showing that minimal contents of these chemical compounds were found in them. This was closely followed by samples with alkali only treated fibers and cement mortars. This is in line with the FTIR results discussed earlier which showed that some peaks disappeared after these two treatments were conducted on the bamboo fibers. Equally, elements such as $\mathrm{Ca}, \mathrm{Fe}, \mathrm{Mg}, \mathrm{S}, \mathrm{C}, \mathrm{Si}$ are associated with cement products and are located in all the samples tested. Most of the surfaces of the samples are coated with tiny layers of cement and the hydrated products in addition to elements found in the bamboo fibers ( $\mathrm{C}$ and $\mathrm{O}$ ), therefore it is expected that a blend of these elements in varying proportions would be found on them.

\section{Discussion}

FTIR analysis of the pre-treated fibers in Fig. 3 clearly showed a sharp contrast in MT system compared with others in the reduction of extractives associated with cellulose fibers. In the same vein, on inclusion with cement and other aggregates in Fig. 4, the chemical composition of the cement composites were changed due to the development of cement hydration products. These were deposited on the fibre surface to fill the voids created by the varying effects of the treatment methods adopted which altered the fibre morphology and architecture. The mechanical strength results in Figs. 5, 6 and 7, indicated that MT treated fibers had more significant effect on the strength than the other treatment meth- ods. This suggests that the good performance recorded for MT was because of improved fiber ductility and greater adhesion of the cement hydrate products to the fibre surface. It has been established through past research experiences and field applications that it is essential for surface of natural fibers to be chemically treated in order to improve the strength, durability and adoption for industrial applications [21]. This conventional method of fiber treatment has been shown to consume a lot of water, chemicals and energy [47]. However, recent emerging studies have revealed that the use of microwave energy in pre-treating natural fibers is an environment friendly option because it saves time needed for treatment which translates to reduced energy cost. Other eco-friendly traits include less heat emitted and it also gives no radiation during operation $[54,55]$. To ease the adoption of this technique in field works, industrial scale microwave devices exist which use magnetrons at $2.45 \mathrm{GHz}$ frequency and it also come with an extra frequency of $900 \mathrm{MHz}$ for fuller range of penetration of the microwave energy [56]. The fibre cement composites produced from this process could be used in the fabrication of lightweight ceiling and roof boards, building materials for low-cost shelters and as wall plasters.

\section{Conclusion}

Cement composites reinforced with bamboo fibers pre-treated with microwave assisted sodium hydroxide, hot water and sodium hydroxide only at $10 \%$ concentration lead to improved mechanical strength due to improved interfacial adhesion between the cement matrix and the fibers. Distinct and better improvement in some properties from microwave assisted sodium hydroxide pre-treated fibers than the alkali only treated fibers were associated with more and effective removal of cellulose, lignin and hemicellulose from the fibers molecular structure which roughened the surface and enabled the cement hydrates to fill the voids leading to good grip and anchorage with the cement matrix. The tensile results for MT showed 24.32 and $20 \%$ higher strength than HT and AA respectively while a slight improvement of AA over HT was noted at $3.6 \%$ improved tensile strength. An higher difference in the bending strength was however observed on comparison between MT and HT with increase in flexural capacity of MT ranging from 54.5 to $97 \%$ in the MOR results and $16-59.1 \%$ for the MOE. The pre-treated fibre cement composites generally showed improved strength on increase of the fibre contents from 1 to $1.5 \%$ for the curing days under consideration. The better performance from the MT and AA treated cementitious composites is also influenced by the compact arrangement of the microstructure coupled with reduced pores and voids on the interface.

\section{Declaration of Competing Interest}

The authors declare that they have no known competing financial interests or personal relationships that could have appeared to influence the work reported in this paper.

\section{Acknowledgement}

The contributions and input from Prof. Chunping Dia of the Department of Wood Science, The British Columbia University, Canada and the anonymous reviewers are greatly appreciated.

\section{Funding}

No funding was received for this study. 


\section{References}

[1] E.J. da Silva, M.L. Marques, F.G. Velasco, C.F. Junior, F.M. Luzardo, M.M. Tashima, A new treatment for coconut fibers to improve the properties of cement-based composites-combined effect of natural latex/pozzolanic materials, Sustainable Mater.Technol. 12 (2017) 44-51.

[2] B.A. Akinyemi, T.E. Omoniyi, Effect of moisture on thermal properties of acrylic polymer modified mortar reinforced with alkali treated bamboo fibres, J. Indian Acad. Wood Sci. 15 (1) (2018) 45-51.

[3] B. Akinyemi, T. Omoniyi, Properties of Latex Polymer Modified Mortars Reinforced with Waste Bamboo Fibers from Construction Waste, Build. 8 (11) (2018) 149.

[4] R.R. Almeida, C.H.S. Del Menezzi, D.E. Teixeira, Utilization of the coconut shell of babaçu (Orbignya sp.) to produce cement-bonded particleboard, Bioresour. Technol. 85 (2) (2002) 159-163.

[5] S.M. Islam, R.R. Hussain, M.A.Z. Morshed, Fiber-reinforced concrete incorporating locally available natural fibers in normal-and high-strength concrete and a performance analysis with steel fiber-reinforced composite concrete, J. Compos. Mater. 46 (1) (2012) 111-122.

[6] R. Sudin, N. Swamy, Bamboo and wood fibre cement composites for sustainable infrastructure regeneration, J. Mater. Sci. 41 (21) (2006) 69176924.

[7] H. Savastano Jr, S.F. Santos, M. Radonjic, W.O. Soboyejo, Fracture and fatigue of natural fiber-reinforced cementitious composites, Cem. Concr. Compos. 31 (4) (2009) 232-243.

[8] R.D. Toledo Filho, F. de Andrade Silva, E.M.R. Fairbairn, J. de Almeida Melo Filho, Durability of compression molded sisal fiber reinforced mortar laminates, Constr. Build. Mater. 23 (6) (2009) 2409-2420.

[9] L. Yan, Effect of alkali treatment on vibration characteristics and mechanical properties of natural fabric reinforced composites, J. Reinf. Plast. Compos. 31 (13) (2012) 887-896.

[10] M. Quintero-Dávila, S.N. Monteiro, H.A. Colorado, Composites of Portland cement and fibers of Guadua angustifolia Kunth from Colombia, J. Compos. Mater. 53 (7) (2019) 883-892.

[11] E.B.C. Santos, C.G. Moreno, J.J.P. Barros, D.A.D. Moura, F.D.C. Fim, A. Ries, L.B.D. Silva, Effect of alkaline and hot water treatments on the structure and morphology of piassava fibers, Mater. Res. 21 (2) (2018).

[12] A. Le Duigou, A. Bourmaud, E. Balnois, P. Davies, C. Baley, Improving the interfacial properties between flax fibres and PLLA by a water fibre treatment and drying cycle, Ind. Crops Prod. 39 (2012) 31-39.

[13] M. Benítez-Guerrero, J. López-Beceiro, P.E. Sánchez-Jiménez, J. Pascual-Cosp. Comparison of thermal behavior of natural and hot-washed sisal fibers based on their main components: Cellulose, xylan and lignin. TG-FTIR analysis of volatile products, Thermochim Acta 581 (2014) 70-86.

[14] A.S. Fonseca, S. Panthapulakkal, S.K. Konar, M. Sain, L. Bufalino, J. Raabe, G.H.D. Tonoli, Improving cellulose nanofibrillation of non-wood fiber using alkaline and bleaching pre-treatments, Ind. Crops Prod. 131 (2019) 203-212.

[15] M. Sood, G. Dwivedi, Effect of fiber treatment on flexural properties of natural fiber reinforced composites A review, Egypt. J. Pet. (2017).

[16] K. Bilba, H. Savastano Junior, K. Ghavami, Treatments of non-wood plant fibres used as reinforcement in composite materials, Mater. Res. 16 (4) (2013) 903-923.

[17] S.R. Ferreira, F. de Andrade Silva, P.R.L. Lima, R.D. Toledo Filho, Effect of hornification on the structure, tensile behavior and fiber matrix bond of sisal, jute and curauá fiber cement based composite systems, Constr. Build. Mater. 139 (2017) 551-561.

[18] J.L. Pehanich, P.R. Blankenhorn, M.R. Silsbee, Wood fiber surface treatment level effects on selected mechanical properties of wood fiber-cement composites, Cem. Concr. Res. 34 (1) (2004) 59-65.

[19] J. Claramunt, M. Ardanuy, J.A. García-Hortal, Effect of drying and rewetting cycles on the structure and physicochemical characteristics of softwood fibres for reinforcement of cementitious composites, Carbohydr. Polym. 79 (1) (2010) 200-205.

[20] Y.F. Huang, P.T. Chiueh, S.L. Lo, A review on microwave pyrolysis of lignocellulosic biomass, Sustainable Environ. Res. 26 (3) (2016) 103-109.

[21] V. Chaturvedi, P. Verma, An overview of key pretreatment processes employed for bioconversion of lignocellulosic biomass into biofuels and value added products. 3, Biotech 3 (5) (2013) 415-431.

[22] C. Yin, Microwave-assisted pyrolysis of biomass for liquid biofuels production, Bioresour. Technol. 120 (2012) 273-284.

[23] M. Guiotoku, F.A. Hansel, E.H. Novotny, C.M.B.D.F. Maia, Molecular and morphological characterization of hydrochar produced by microwaveassisted hydrothermal carbonization of cellulose, Pesquisa Agropecuária Brasileira 47 (5) (2012) 687-692.

[24] Akinyemi, B. A., Bamidele, A., \& Joel, E. Response of coir fibre reinforced cement composites to water repellent chemical additive and microwave accelerated curing. Cellulose, 1-13.

[25] P. Verma, T. Watanabe, Y. Honda, T. Watanabe, Microwave-assisted pretreatment of woody biomass with ammonium molybdate activated by $\mathrm{H}_{2} \mathrm{O}_{2}$, Bioresour. Technol. 102 (4) (2011) 3941-3945.

[26] D.L. Gil-López, J.A. Lois-Correa, M.E. Sánchez-Pardo, M.A. Domínguez-Crespo, A.M. Torres-Huerta, A.E. Rodríguez-Salazar, V.N. Orta-Guzmán, Production of dietary fibers from sugarcane bagasse and sugarcane tops using microwaveassisted alkaline treatments, Ind. Crops Prod. 135 (2019) 159-169.
[27] Z. Zhu, R. Simister, S. Bird, S.J. McQueen-Mason, L.D. Gomez, D.J. Macquarrie, Microwave assisted acid and alkali pretreatment of Miscanthus biomass for biorefineries, matrix 10 (2015) 16.

[28] L. Zhou, V. Budarin, J. Fan, R. Sloan, D. Macquarrie, Efficient method of lignin isolation using microwave-assisted acidolysis and characterization of the residual lignin, ACS Sustainable Chem. Eng. 5 (5) (2017) 3768-3774.

[29] I. Janker-Obermeier, V. Sieber, M. Faulstich, D. Schieder, Solubilization of hemicellulose and lignin from wheat straw through microwave-assisted alkali treatment, Ind. Crops Prod. 39 (2012) 198-203.

[30] G.G. Allan, B.B. Krieger, D.W. Work, Dielectric loss microwave degradation of polymers: cellulose, J. Appl. Polym. Sci. 25 (9) (1980) 1839-1859.

[31] Dullah, H., Abidin Akasah, Z., Zaini Nik Soh, N. M., \& Mangi, S. A. (2017). Compatibility improvement method of empty fruit bunch fiber as a replacement material in cement bonded boards: A review. In Materials Science and Engineering Conference Series (Vol. 271, No. 1, p. 012076).

[32] J. Kasim, A.J. Ahmad, A.L. Mohamod, K.C. Khoo, A note on the proximate chemical composition and fibre morphology of Bambusa vulgaris, J. Trop. For. Sci. 6 (3) (1994) 356-358.

[33] O. Agu, L. Tabil, T. Dumonceaux, Microwave-assisted alkali pre-treatment, densification and enzymatic saccharification of canola straw and oat hull, Bioengineering 4 (2) (2017) 25.

[34] L.Y. Mwaikambo, M.P. Ansell, Chemical modification of hemp, sisal, jute, and kapok fibers by alkalization, J. Appl. Polym. Sci. 84 (12) (2002) 2222-2234.

[35] Kovacevic, Z., Bischof, S., Vujasinovic, E., \& Fan, M. (2016). The influence of pretreatment of Spartium junceum L. fibres on the structure and mechanical properties of biocomposites.

[36] Pao, C. N. Z., \& Yeng, C. M. (2018). Properties and characterization of wood plastic composites made from agro-waste materials and post-used expanded polyester foam. Journal of Thermoplastic Composite Materials, 0892705718772877.

[37] D. Ray, B.K. Sarkar, Characterization of alkali treated jute fibers for physical and mechanical properties, J. Appl. Polym. Sci. 80 (7) (2001) 1013-1020.

[38] M.S. Islam, K.L. Pickering, N.J. Foreman, Influence of alkali fiber treatment and fiber processing on the mechanical properties of hemp/epoxy composites, J. Appl. Polym. Sci. 119 (6) (2011) 3696-3707.

[39] Adefisan, O. O., Fabiyi, J. S., \& McDonald, A. G. (2012). Hydration behaviour and infrared spectroscopy of pre-treatments effects on portland cementeremospatha macrocarpa and laccosperma secundiflorum systems.

[40] S. Chakraborty, S.P. Kundu, A. Roy, B. Adhikari, S.B. Majumder, Effect of jute as fiber reinforcement controlling the hydration characteristics of cement matrix, Ind. Eng. Chem. Res. 52 (3) (2013) 1252-1260.

[41] B.W. Jo, S. Chakraborty, A mild alkali treated jute fibre controlling the hydration behaviour of greener cement paste, Sci. Rep. 5 (2015) 7837.

[42] A.A. Mohammed, D. Bachtiar, M.R.M. Rejab, J.P. Siregar, Effect of microwave treatment on tensile properties of sugar palm fibre reinforced thermoplastic polyurethane composites, Defence Technol. 14 (4) (2018) 287-290.

[43] A.K. Mohanty, M. Misra, L.T. Drzal, Surface modifications of natural fibers and performance of the resulting biocomposites: an overview, Compos. Interfaces 8 (5) (2001) 313-343.

[44] Joseph, K., Mattoso, L. H. C., Toledo, R. D., Thomas, S., De Carvalho, L. H., Pothen, L. \& James, B. (2000). Natural fiber reinforced thermoplastic composites. Natural polymers and agrofibers composites, 159.

[45] Kovačević, Z., Bischof, S., Vujasinović, E., \& Fan, M. (2016). The influence of pretreatment of Spartium junceum L. fibres on the structure and mechanical properties of PLA biocomposites. Arabian Journal of Chemistry.

[46] A. Orue, A. Jauregi, U. Unsuain, J. Labidi, A. Eceiza, A. Arbelaiz, The effect of alkaline and silane treatments on mechanical properties and breakage of sisal fibers and poly (lactic acid)/sisal fiber composites, Compos. A Appl. Sci. Manuf. 84 (2016) 186-195.

[47] S.i. Mistik, E.D. Kocak, N. Merdan, Effect of the Ecological Methods on the Surface Modification of the Kenaf Fibers, Mater. Sci. 22 (3) (2016) 409-414.

[48] O.A. Hisseine, W. Wilson, L. Sorelli, B. Tolnai, A. Tagnit-Hamou, Nanocellulose for improved concrete performance: A macro-to-micro investigation for disclosing the effects of cellulose filaments on strength of cement systems, Constr. Build. Mater. 206 (2019) 84-96.

[49] M.C.N. Yemele, A. Koubaa, A. Cloutier, P. Soulounganga, T. Stevanovic, M.P. Wolcott, Effects of hot water treatment of raw bark, coupling agent, and lubricants on properties of bark/HDPE composites, Ind. Crops Prod. 42 (2013) $50-56$.

[50] A. Guo, O.B. Aamiri, J. Satyavolu, Z. Sun, Impact of thermally modified wood on mechanical properties of mortar, Constr. Build. Mater. 208 (2019) 413-420.

[51] E.T. Dawood, M. Ramli, Development of high strength flowable mortar with hybrid fiber, Constr. Build. Mater. 24 (6) (2010) 1043-1050.

[52] M. Li, S. Zhou, X. Guo, Effects of alkali-treated bamboo fibers on the morphology and mechanical properties of oil well cement, Constr. Build. Mater. 150 (2017) 619-625.

[53] R. Malenab, J. Ngo, M. Promentilla, Chemical treatment of waste abaca for natural fiber-reinforced geopolymer composite, Mater. 10 (6) (2017) 579.

[54] L.D. Dias, K.H. Gonçalves, J.E. Queiroz, G.M. Vila Verde, G.L. Aquino, An ecofriendly and alternative method of forced degradation of fluoroquinolone drugs by microwave irradiation: a new application for analytical eco-scale, J. Microw. Power Electromagn. Energy. 52 (3) (2018) 162-181.

[55] W.L.E. Wong, M. Gupta, Using microwave energy to synthesize light weight/ energy saving magnesium based materials: a review, Technologies 3 (1) (2015) 1-18.

[56] R. Prommas, T. Rungsakthaweekul, Effect of microwave curing conditions on high strength concrete properties, Energy Procedia 56 (2014) 26-34. 